536. $46: 662.612$

\title{
A Study of Combustion in Simple Combustion Chamber*
}

\author{
By Ryūichi Matsumoto**, Куојі Кгмото***, \\ and Akiyo FUKUWATARI****
}

\begin{abstract}
This paper deals with the low frequency type of oscillations in a simple combustion chamber where the fuel used is propane. The phenomenon was found to be closely connected with the progressive flame necking from the results of flame photographs by a high speed camera and the frequencies of oscillations were able to be calculated as a Helmholtz resonator. From both results mentioned above and measurements of pressure drop along the combustion chamber, it was found that the oscillations were caused by the negative gradient of the characteristic curve which represents the relation between the pressure drop and the secondary air velocity in the vicinity of the flame necking. Some theoretical analyses were made and a differential equation of Van der Pol type was obtained. The analytical values of the frequencies well agreed with the experimental results and an example of oscillation patterns on phase-plane was in good agreement with that of oscillograms.
\end{abstract}

\section{Introduction}

Recently it is known that the combustion instabilities are possible in different two modes of oscillations. One of them is an acoustic type of oscillations characterized by high frequency, i.e. high-frequency oscillation, and the other is a nonacoustic type of oscillations, i.e. low-frequency oscillation. The low-frequency oscillation leads to troubles such as blow-out, low combustion efficiency and so on. But it seems that there are not so many reports about this oscillation except several works: such as studies in rocket motor by L. Crocco et 'al. (1), in boilers by M. W. Thring ${ }^{(2)}$ and F. Mauss et al. ${ }^{\left({ }^{3}\right)}$, and a study of simple combustion chamber by Sato et al. (4) These studies indicate that the combustion instabilities are caused by the combustion time lag, but it is known that the combustion instabilities are not always dependent upon its time lag in a few studies $^{(5) \sim(8)}$ using some gaseous fuels. For example, Nishimura ${ }^{(5)}$ deals with the combustion oscillations in a simple combustion chamber using hydrogen gas, and shows that the combustion instabilities are caused by the temperature fluctuation of the burnt gas at the outlet of combustion chamber. Thus, it seems that there are many causes to generation of the oscillations for various kinds of

* Received 9 th August, 1967.

** Professor, Faculty of Engineering, Kobe University, Nada-ku, Kobe.

*** Assistant, Faculty of Engineering, Kobe University.

***** Post Graduate Student, Faculty of Engineering, Kobe University. conditions.

This paper deals with the low-frequency oscilla. tion using a gaseous fuel (propane) in a simple combustion chamber. It is found that the phenomenon occurs in the vicinity of the progressive flame necking with the increase of the secondary air velocity, and that the region with a stable combustion becomes very narrow by reason of this phenomenon. Such a phenomenon is also reported by J. Barr ${ }^{(9)}$. who carried out an experimental study of combustion using butane gas as a fuel.

\section{Nomenclature}

$A$ : cross sectional area of combustion chamber

$B$ : flow rate of the secondary air

$F: V_{0} / \kappa \bar{P}_{0}$ defined in Eq. (1)

$H$ : total pressure drop, $H=P_{0}-P_{2}$

$L$ : length of combustion chamber

$M$ : air-fuel ratio

$N: \gamma_{m} L / g$ defined in Eq. (9)

$P$ : pressure

$\Delta P:$ pressure drop

$P_{r}$ : frictional loss in combustion chamber

$Q_{\alpha 1}$ : flow rate of the primary air

$Q_{f}:$ flow rate of fuel

$T:$ temperature

$U:$ secondary air velocity in combustion chamber

$U_{a_{1}+f}:$ mixture velocity of fuel and the primary air

$V: V=U_{1} / \sqrt{k}$

$V_{0}:$ volume of surge tank

$a(u): a(u)=d \varphi(u) / d u$ defined in Eq. (13) 
$c:$ acoustic velocity

$d$ : inner diameter of combustion chamber

$f$ : frictional factor defined in Eq. (2)

$g:$ acceleration of gravity

$h$ : fluctuational component of total pressure drop

$k: k=F / A N$ defined in Eq. (15)

$t:$ time

$u$ : fluctuational component of velocity

$v: v=u / \sqrt{k}$ defined in Eq. (16)

$\gamma:$ specific weight

$\theta$ : angle

$\kappa:$ ratio of specific heat

$\lambda$ : frictional factor in combustion chamber defined in Eq. (4)

$\nu$ : frequency

$\rho:$ density

$\phi\left(\bar{U}_{1}\right)$ : characteristic curve which represents relation between pressure drop and the secondary air velocity

$\omega$ : angular velocity

Subscripts

0 : value in surge tank

1 : value at inlet of combustion chamber

$2:$ value at outlet of combustion chamber

$a$ : primary air

$f:$ fuel

$m:$ mean value in combustion chamber

- : time mean value

\section{Experimental apparatus and procedure}

The arrangement of the apparatus is shown in Fig. 1. Propane-gas (purity $95 \%$ ) and the primary air are mixed perfectly in the mixing chamber (1). Then, the mixture is jetted from the burner pipe (2) and fired in the combustion chamber (3). The secondary air is introduced into the combustion chamber through the surge tank (4) from the blower, and its flow rate is measured by an oriffice (7) and a manometer (8). The static pressures at the outlet of blower and at the inlet of surge tank are respectively measured by manometers (9) and (10). The

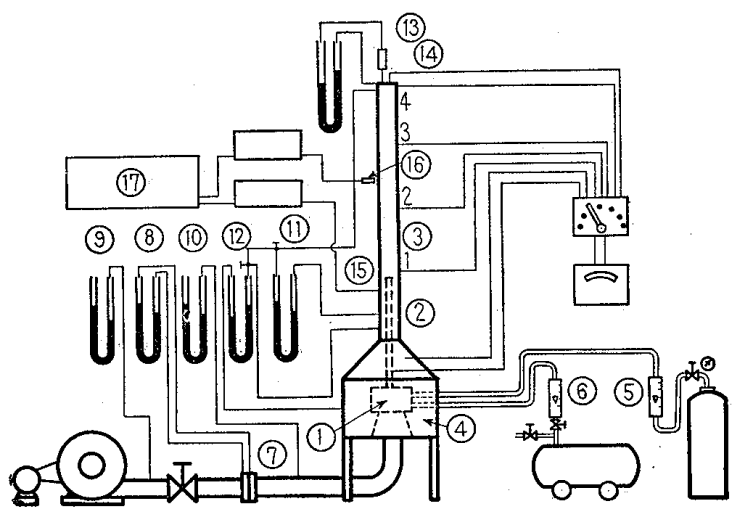

Fig. 1: Experimental apparatus pressure drop in the combustion chamber (pressure difference between the value at the inlet and at the outlet of combustion chamber), is measured by manometer (11) and the pressure drop in passing through the surge tank (pressure difference between the value at the surge tank and at the inlet of combustion chamber) and the total pressure drop (pressure difference between the value at the surge tank and at the outlet of combustion chamber) are measured by manometer (12).

The burnt gas velocity at the outlet of combustion chamber is measured by a Pitot-tube (13). The temperature of the burnt gas is measured by alumelchrommel thermocouple (14). Further, the temperature of air at the inlet of combustion chamber, the value of mixture in the burner pipe and the outer wall temperature along the combustion chamber are also measured by alumel-chrommel thermocouples. The fluctuations of the secondary air velocity and flame are respectively recorded on the oscillograph (17) by the hot wire anemometer (15) and the photo-transister (16).

With the selected values of air-fuel ratio $M$ and fuel flow rate $Q_{f}$, the experimental works are carried out by changing the secondary air velocity $U_{1}$ and the mixture velocity $U_{a_{1}+f}$. In this experiment, observing the movement of flame, the static pressure, pressure drop and temperarure are also measured. Moreover, the lower limit of the secondary air velocity sufficient to produce a low-frequency oscillation (it is called the oscillation limit in the following) is measured and the factors affecting the oscillation limit are examined by changing the mixture velocity, the secondary air velocity, the air-fuel ratio and the geometrical factors of the system such as the length and the cross-sectional area of combustion chamber and the volume of surge tank. At the same time, these effects on frequencies are examined, too. The burner pipe used in this study is made of brass and has a thickness of $2.95 \mathrm{~mm}$, inner diameter of $10 \mathrm{~mm}$ and length of $400 \mathrm{~mm}$.

\section{Experimental results and discussion}

4.1 Flame movement owing to the increment of the secondary air velocity

Figure 2 shows a series of photographs of the appearance of flame movement owing to the increment of the secondary air velocity. In this case, a pyrex glass pipe is used for combustion chamber. These pictures show that the flame length becomes shorter and the state of combustion changes from a stable combustion to an acoustic oscillation and finally leads to a low-frequency oscillation with an increase of the secondary air velocity. Moreover, it is found from the observation of flame movement that just before the low-frequency oscillation is 


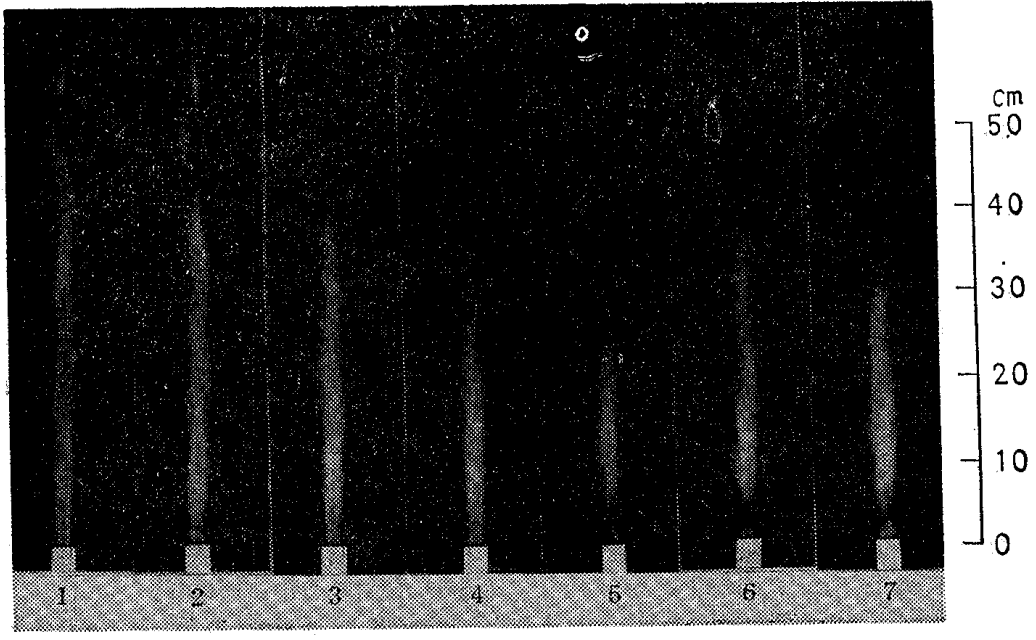

\begin{tabular}{|c|c|c|c|c|}
\hline & State of combustion & Observed character of flame & $\overline{U_{1}} \mathrm{~m} / \mathrm{sec}$ & Oscillogram \\
\hline 1 & Stable & $\begin{array}{l}\text { Dead space of about } 1 \mathrm{~mm} \text { is observed between } \\
\text { flame and the burner tip. }\end{array}$ & 14.6 & (A) \\
\hline 2 & Stable & Pale blue flame & 15.8 & \\
\hline 3 & Acoustic oscillation & $\begin{array}{l}\text { Sometimes resonance sound by acoustic oscillation } \\
\text { is heard. }\end{array}$ & 17.0 & \\
\hline 4 & Acoustic oscillation & Resonance sound is heard intensively. & 18.0 & (B) \\
\hline 5 & $\begin{array}{l}\text { Transitional state from acoustic oscil- } \\
\text { lation to low-frequency oscillation }\end{array}$ & $\begin{array}{l}\text { Periodic flame necking is recognized at the point } \\
\text { about } 10 \mathrm{~mm} \text { upward from the burner tip. }\end{array}$ & 18.6 & \\
\hline 6 & $\begin{array}{l}\text { A picture just before the low-frequency } \\
\text { oscillation is generated }\end{array}$ & same as 5 & 18.8 & . \\
\hline 7 & Low-frequency oscillation & A picture during low-frequency oscillation & 19.0 & (D) \\
\hline
\end{tabular}

$d=65 \mathrm{~mm}, M=8, Q_{f}=4.6 \mathrm{l} / \mathrm{m}, Q_{a 1}=56.0 \mathrm{l} / \mathrm{m}$

Fig. 2 Flame movement owing to the increment of the secondary air velocity

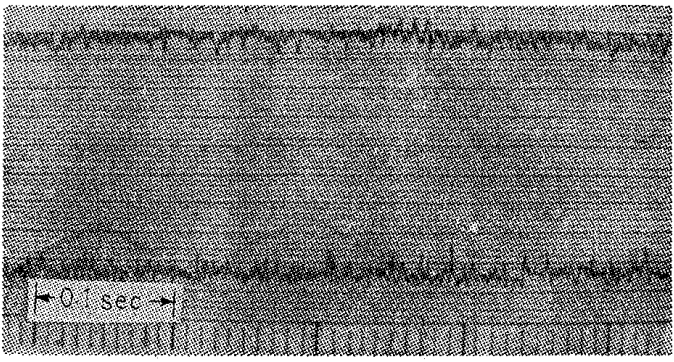

(A)

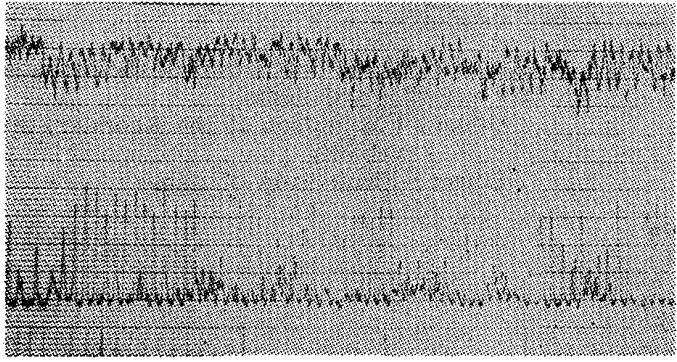

(B)

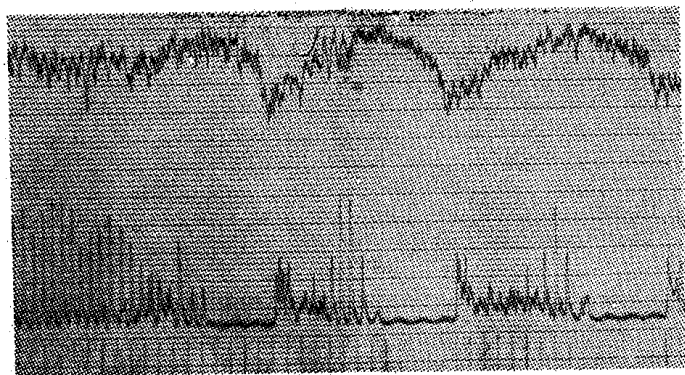

(C)

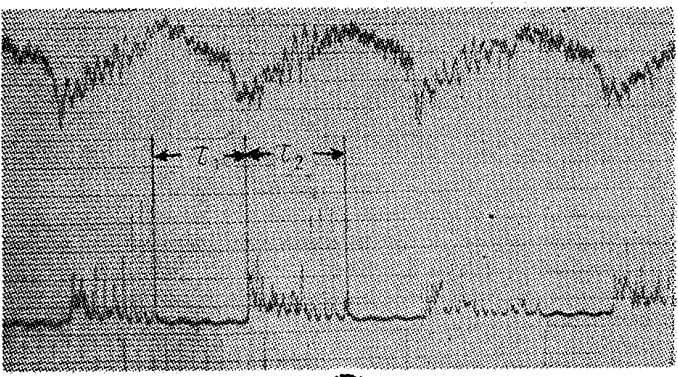

(D)

Upper : Fluctuation of the secondary air Lower: Fluctuation of flame $\tau_{1}$ : Duration of blow-out $\tau_{2}$ : Duration of burning $d=49.5 \mathrm{~mm}, \quad M=8, \quad Q_{f}=4.6 \mathrm{l} / \mathrm{m}, \quad Q_{a 1}=56.0 \mathrm{l} / \mathrm{m}$

(A), (B) and (D) are respectively corresponding to the key words in Fig. 2, and (C) is a transitional state from the flame necking to the low-frequency oscillation. Frequency (c/sec): (B) 147 , (D) 7.6

Fig. 3 Typical oscillograms corresponding to each state of flame movement 
generated, a convergent part of flame (i.e. flame necking) is recognized at the point about $10 \mathrm{~mm}$ upward from the burner tip. Typical oscillograms corresponding to each state of flame movement are shown in Fig. 3. Figure 3 (C) shows a transitional state from the flame necking to the low-frequency oscillation. In this figure, it is found that the generation of the low-frequency oscillation is caused mainly by the fluctuation of the secondary air velocity.

\subsection{Mechanism of low-frequency oscillation}

4.2.1 A Series of photographs of low-frequency oscillation by high speed camera

Figure 4 shows a series of flame photographs of the low-frequency oscillation by a high speed camera (900 frames / sec). The figure of the right hand side in Fig. 4 shows a model for the fluctuation of the secondary air velocity and of flame. The photograph 1 shows a small flame on the burner tip. As the fuel is fired by this small flame, the flame front flows downstream with an increase of the secondary air velocity $2 \sim 8$ and reaches the state of the longest flame 9. Then, the necking is generated near the bottom of flame and finally: the flame disappears except a small flame at the burner tip 18. After several milli-seconds of duration of blow-out, the state of flame returns to the original state 1.

\subsubsection{Empirical facts}

The basic empirical facts about the low-frequency oscillation are as follows:

(1) The oscillation limit is independent of the length of combustion chamber and the volume of surge tank, but it is determined by the values of air-fuel ratio, the mixture velocity and the secondary air velocity. Figure 5 shows the oscillation limits in the case of each air-fuel ratio using a thickness of $2.95 \mathrm{~mm}$ for the burner tip. But when a different value of thickness of the burner tip is used, it must be pointed out that the values of the oscillation limits are also remarkably varied from the results obtained above.

(2) The frequencies are not so much influenced by air-fuel ratio, the mixture velocity and the secondary air velocity.

(3) Figures 6, 7 and 8 show that the experi. mental results of the frequencies are varied by the length and the cross-sectional area of combustion chamber or the volume of surge tank. The values of Helmholtz-frequencies are shown by solid and broken lines and these are analyzed later.

(4) The frequencies are not influenced by the length of the burner.

(5) When the inlet of combustion chamber is restricted with a throttle, the oscillation is not sustained, because the oscillation which is generated in the combustion chamber does not influence the surge tank.

(6) Even if the burner pipe is restricted with a throttle, the generated oscillation is sustained.
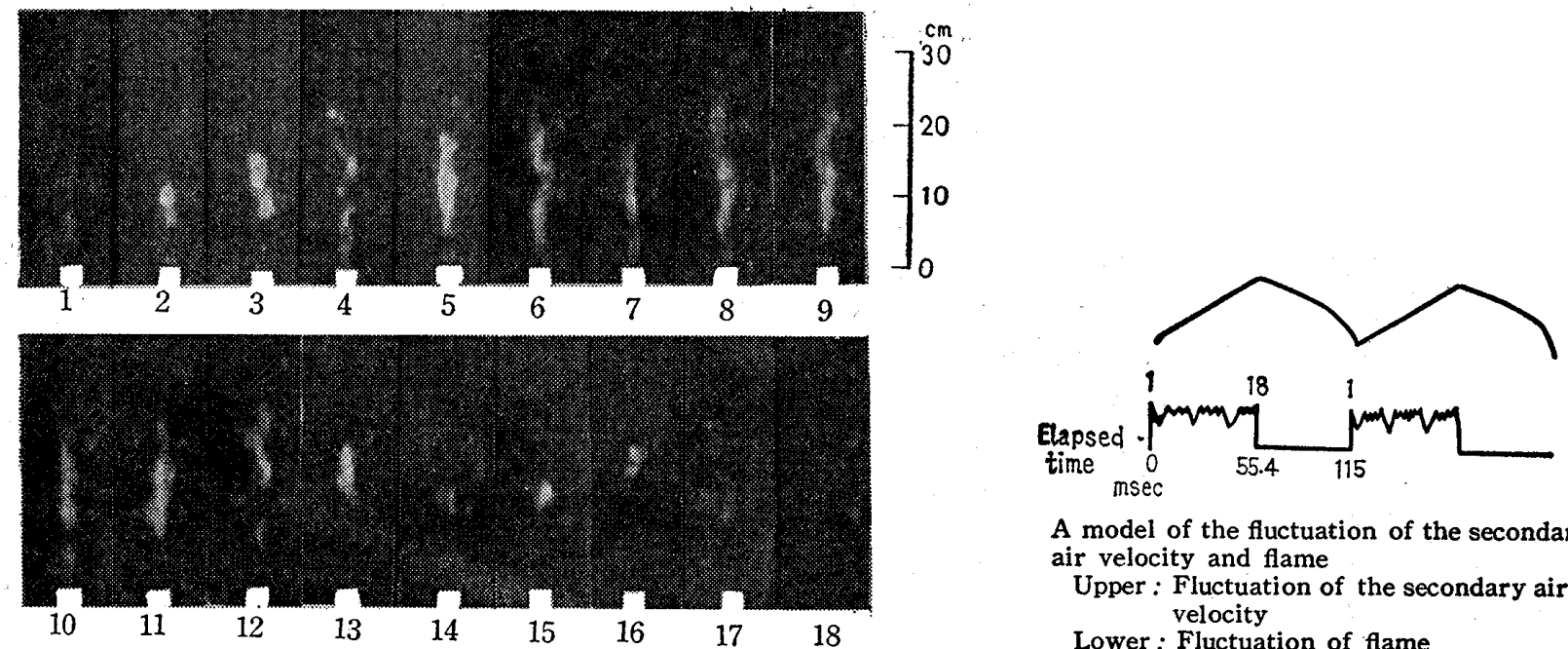

A model of the fluctuation of the secondary air velocity and flame

Upper : Fluctuation of the secondary air velocity

Lower : Fluctuation of flame

\begin{tabular}{c|c|c|c|c|c|c|c|c|c|c}
\hline No. of photos & 1 & 2 & 3 & 4 & 5 & 6 & 7 & 8 & 9 & 10 \\
\hline $\begin{array}{c}\text { Elapsed time } \\
\text { msec }\end{array}$ & 0 & 1.8 & 3.6 & 6.3 & 10.4 & 14.5 & 18.6 & 22.7 & 26.8 & 30.9 \\
\hline \begin{tabular}{c} 
No. of photos \\
\hline $\begin{array}{c}\text { Elapsed time } \\
\text { msec }\end{array}$
\end{tabular} & 11 & 12 & 13 & 14 & 15 & 16 & 17 & 18 & $\cdots$ & 1 \\
\hline
\end{tabular}

$\therefore: \quad d=65 \mathrm{~mm}, \quad M=8, Q_{\rho}=4.6 \mathrm{l} / \mathrm{m}, Q_{a 1}=56.0 \mathrm{l} / \mathrm{m}, \quad \bar{U}_{1}=19.4 \mathrm{~m} / \mathrm{sec}$

Fig: 4...A series of photographs of low-frequency oscillation by high speed camera 
(7) From the photographs by a high speed camera, it is recognized that the flame is swept away downstream with the velocity about $22 \mathrm{~m} / \mathrm{sec}$ and this velocity is almost the same value as the secoondary air velocity.

Considering these facts mentioned above, it is found that the generation of oscillation is mainly

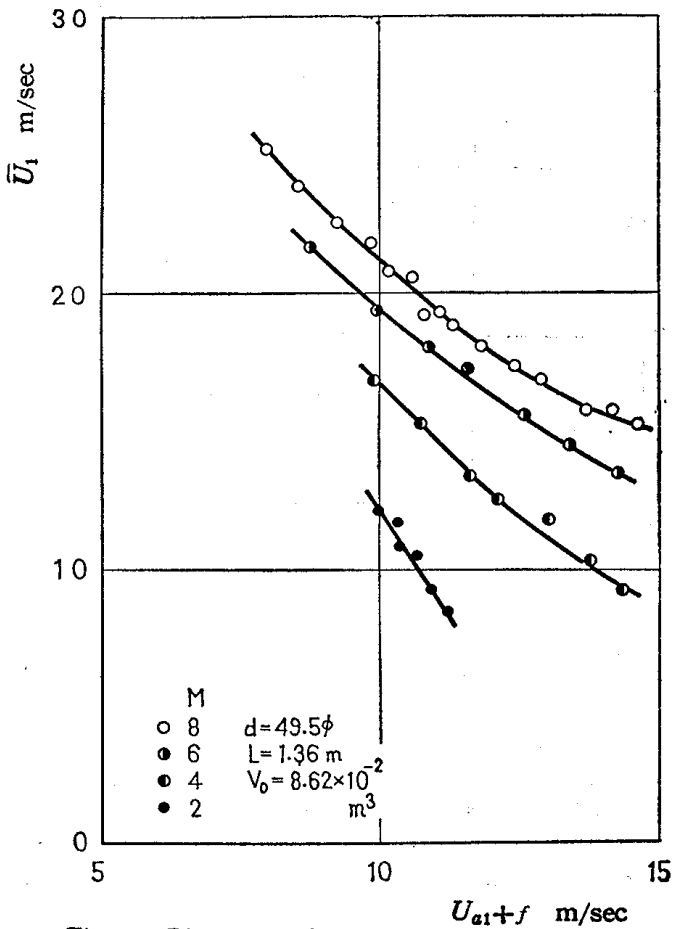

Fig. 5 Diagram of lower oscillation limit

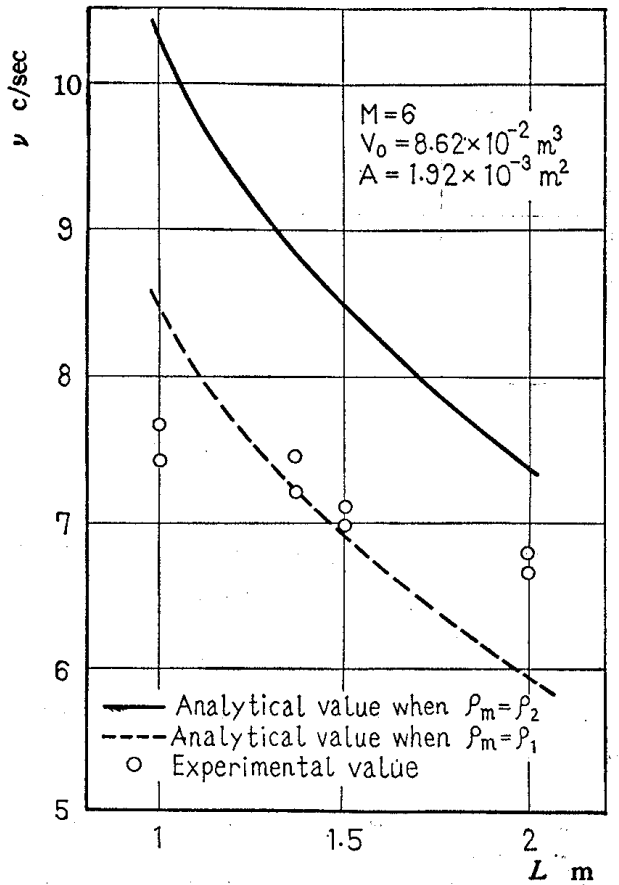

Fig. 6 Effect of the length of combustion chamber on frequency caused by the fluctuation of the secondary air velocity and the surge tank plays an important part for the duration of the oscillation, and the frequency is determined by the geometrical factors of the apparatus. Moreover, from the observation of flame in Fig. 2, it is seen that the flame necking occurs in the vicinity of the oscillation limit.

4.2.3 Discussion on mechanism of oscillation.

From the results mentioned above, it seems that the main cause of oscillation is the fluctuation of the secondary air velocity when the flame necking appears, and that the fluctuation of flame is a result of resonance with that of the secondary air velocity.

Now, the mechanism of generation of oscillation is explained in the following.

Assuming that the flow in the combustion chamber is a one-dimensional flow with heat genera-

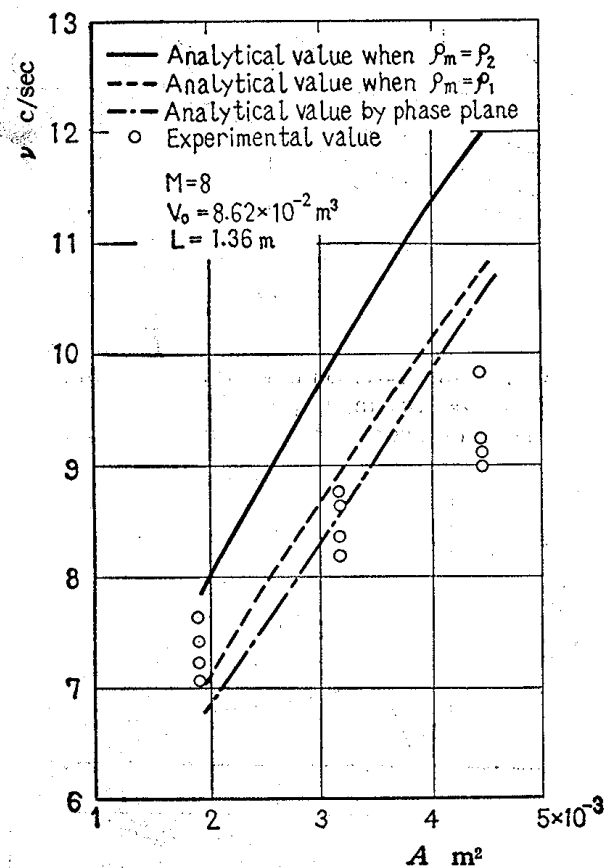

Fig. 7 Effect of the cross-sectional area of combustion chamber on frequency

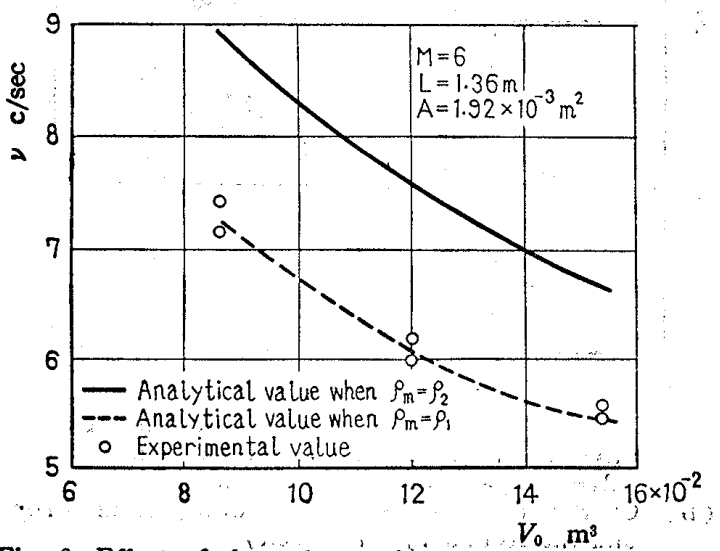

Fig. 8 Effect of the volume of surge tank on frequency 
tion, the value of pressure drop in the combustion chamber is the sum of frictional loss, accelerational loss and loss of head. Among them, the frictional

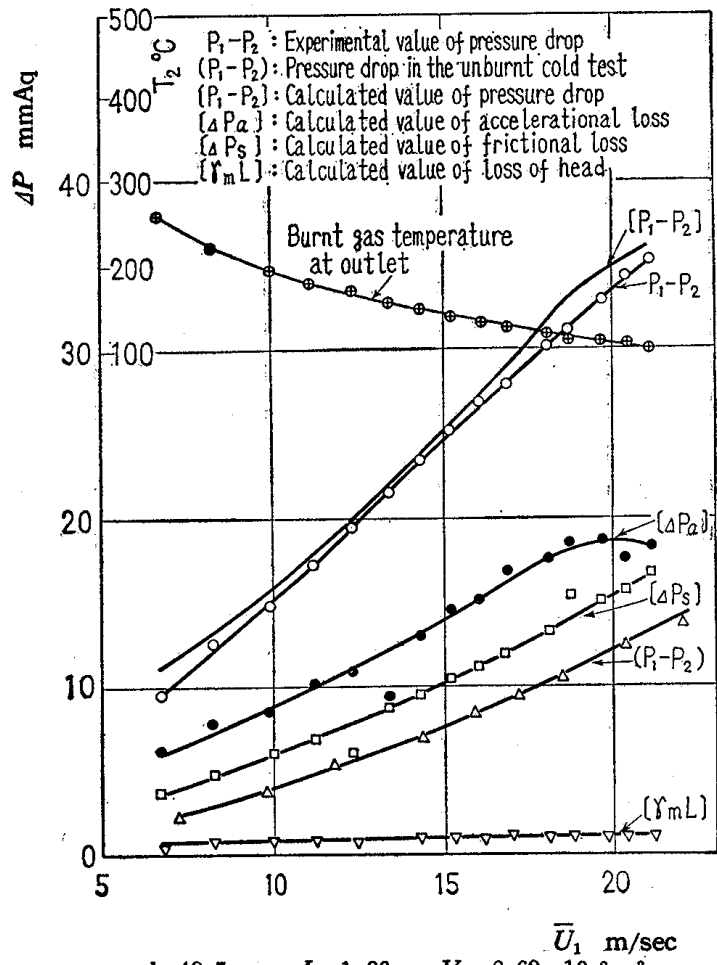

$d=49.5 \mathrm{~mm}, L=1.36 \mathrm{~m}, V_{0}=8.62 \times 10^{-2} \mathrm{~m}^{3}$, $M=6, Q_{f}=3.0 \mathrm{l} / \mathrm{m}, Q_{a}=27.6 \mathrm{l} / \mathrm{m}$

(a) Pressure drop in the combustion chamber

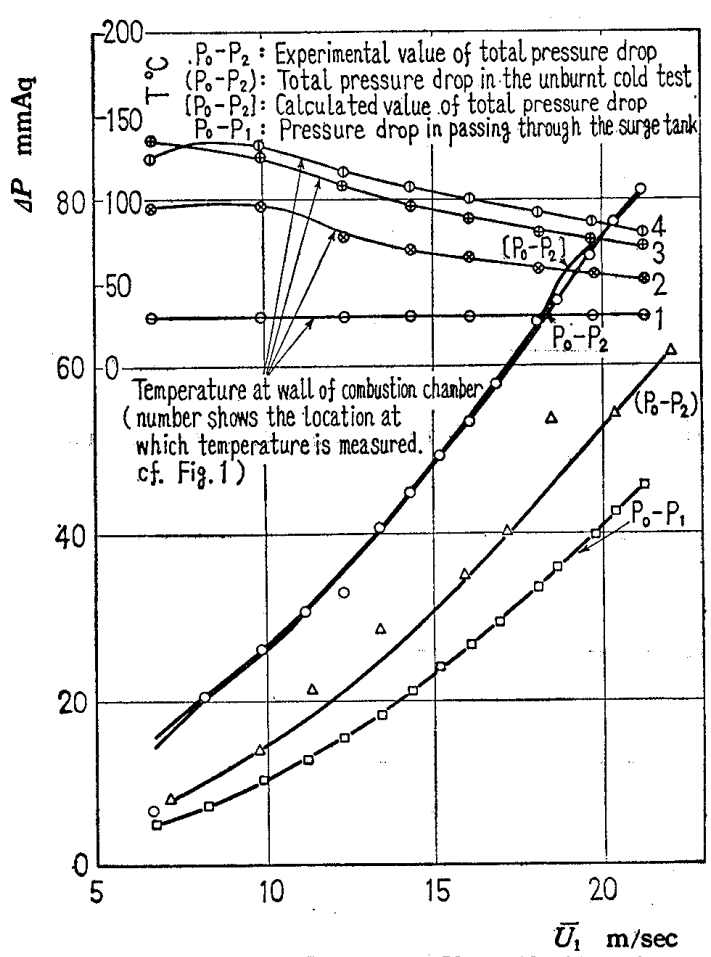

$d=49.5 \mathrm{~mm}, L=1.36 \mathrm{~m}, V_{0}=8.62 \times 10^{-2} \mathrm{~m}^{3}$

$M=6, Q_{f}=3.0 \mathrm{l} / \mathrm{m}, Q_{a 1}=27.8 \mathrm{l} / \mathrm{m}$

(b) Characteristic curve between total pressure drop and the secondary air velocity

Fig. 9 loss increases gradually with an increase of the secondary air velocity, but the accelerational loss is the rate of the increment of momentum by the com-

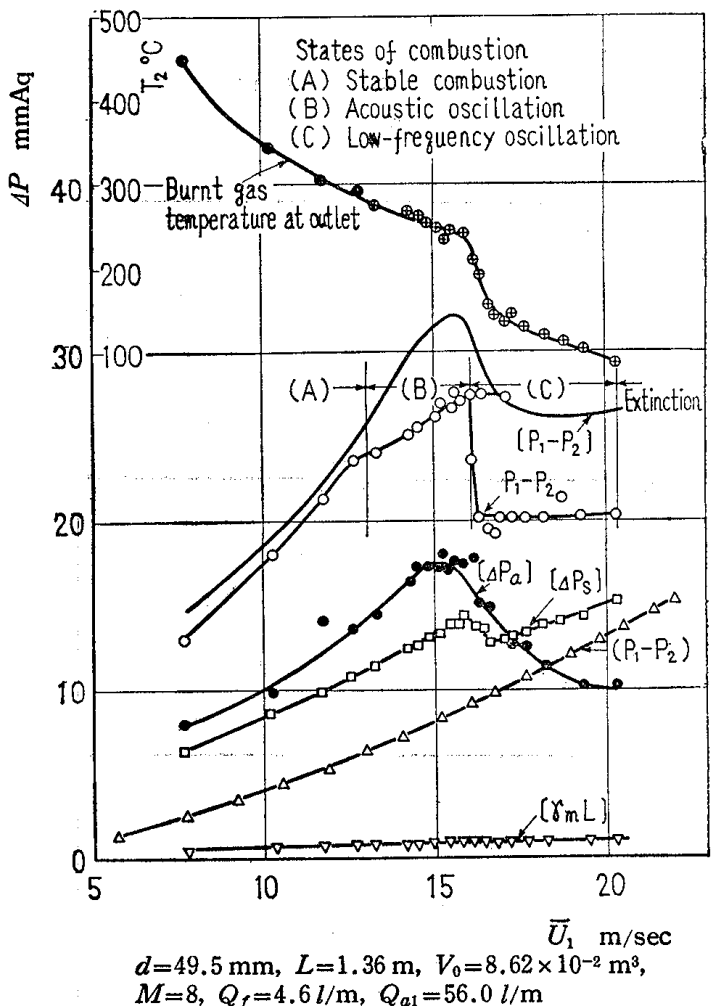

(a) Pressure drop in the combustion chamber

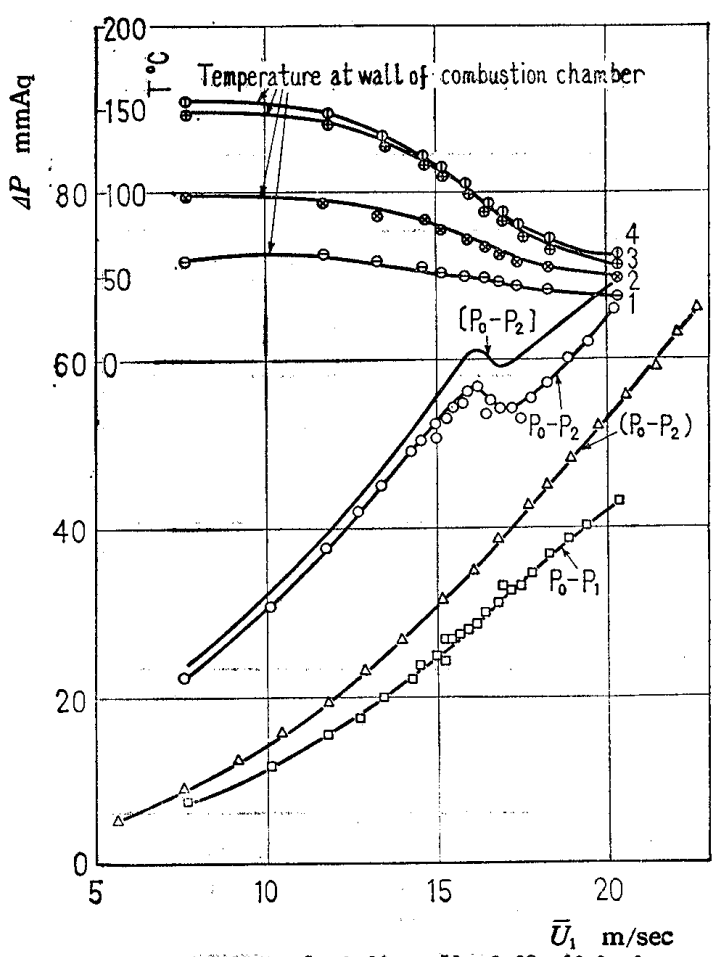

$d=49.5 \mathrm{~mm}, L=1.36 \mathrm{~m}, V_{0}=8.62 \times 10^{-2} \mathrm{~m}^{3}$,

$M=8, Q_{f}=4.6 \mathrm{l} / \mathrm{m}, Q_{a 1}=56.0 \mathrm{l} / \mathrm{m}$

(b) Characteristic curve between total pressure drop and the secondary air velocity 
bustion and its value is determined by the state of combustion. That is, it does not always increase owing to the increment of the secondary air velocity when the flame necking appears. Because in that case, the state of combustion is remarkably poor [See Fig. 10 (a), the burnt gas temperature at the outlet of combustion chamber]. Moreover, it seems that the flame fluctuation shows a steady cycle with a constant period and amplitude, which consists of the duration of burning and blow-out as shown in Fig. 3 (D). From these facts, it seems that the phenomenon is a self-excited oscillation which is based on a character of the pressure drop in the combustion chamber in the vicinity of the flame necking. In Fig. 3 (C), it is obviously noticed that the flame sustained behind the burner tip shows the necking phenomenon and then reaches blow-out with a small increase of the secondary air velocity. The phenomenon grows up to a self-excited oscillation. Thus, it can be seen that this is similar to a surging of a pump or blower. But by sustaining of the constant values of delivery flow rate at the outlet of the blower during the low-frequency oscillation, it is explained clearly that the oscillation is not caused by a surging of blower.

\subsection{Experimental results of pressure drop}

The experimental results of the pressure drop are shown in Figs. 9 and 10 . Figure 9 shows an example when no oscillations are generated even for a high velocity. Figure 10 shows an example when the low-frequency oscillation is caused. Figure 10 (a) shows that the pressure drop in the combustion chamber increases owing to the increment of the secondary air velocity in the A region, and if the high-frequency oscillation appears, the gradient of the curve of the pressure drop becomes gentler in the $B$ region. Furthermore, for the higher value of the secondary air velocity than that in the $\mathrm{B}$ region, the low-frequency oscillation is generated in the $\mathrm{C}$ region where the flame necking is recognized. If the secondary air velocity is more increased, the flame is blown out entirely except a small flame at the burner tip (i.e. extinction). Figure 10 (b) shows the relation between the total pressure drop and the secondary air velocity (the curve is called the characteristic curve in this paper). This characteristic curve is used in order to analyze the oscillation pattern, which is explained later.

\section{Theoretical approach}

\subsection{Fundamental equation}

Analytical model is shown in Fig. 11. In this paper, the following assumptions are made:

(1) The flow rate from blower to surge tank has a constant value. Moreover, the air and the burnt gas obey the law of a perfect gas, respectively.

(2) The flow motion from surge tank to combustion chamber is an adiabatic change.

(3) The mixture flow rate is neglected, because it is much smaller than the secondary air flow rate, and the flow in the combustion chamber is a one-dimensional flow with heat generation.

(4) The combustion chamber can be treated as a concentrated volume system, because it is sufficiently short in comparison with each wave length of the pressure and the velocity,

Under these assumptions, the mechanism of oscillation is explained as follows using an analytical model as shown in Fig. 11.

The change of the pressure in the surge tank is from the assumption (2):

$$
F \frac{d P_{0}}{d t}=B_{0}-B_{1}(t)=B_{0}-A U_{1}
$$

Here, $F=V_{0} / \kappa \vec{P}_{0}$.

By the assumption ( 1 ), $B_{0}=$ const. and the pressure drop in passing through the surge tank is as follows :

$$
P_{0}-P_{1}=f \frac{\gamma_{1}}{2 g} U_{1}^{2}
$$

The pressure drop per unit length along the combustion chamber is

$$
-\frac{\partial P}{\partial x}=\frac{r}{g}\left\{U \frac{\partial U}{\partial x}+\frac{\partial U}{\partial t}\right\}+r+\frac{\partial P_{r}}{\partial x}
$$

Here, $P_{r}$ is mainly a frictional loss at wall and partially contains the mixing loss between the mixture and the secondary air, and so on. $P_{r}$ is described in the same form as Darcy's formula

$$
\frac{\partial P_{r}}{\partial x}=\lambda \frac{\gamma}{2 g} \frac{U^{2}}{d}
$$

Under the assumption (4), Eq. ( 3 ) can be integrated (10). Then considering $\gamma U=\gamma_{1} U_{1}=\gamma_{2} U_{2}$, the following equation is obtained,

$$
\begin{aligned}
P_{1}-P_{2}= & \frac{1}{g}\left(\gamma_{2} U_{2}{ }^{2}-\gamma_{1} U_{1}{ }^{2}\right)+\frac{\gamma_{m} L}{g} \frac{d U_{1}}{d t}+\gamma_{m} L \\
& +\lambda \frac{L\left(\gamma_{1} U_{1}\right)^{2}}{2 g d}\left(\frac{1}{\gamma}\right)_{m} \ldots \ldots \ldots \ldots \ldots \ldots \ldots \ldots \ldots \ldots \ldots \ldots \ldots
\end{aligned}
$$

Here, from the assumption (4),

$$
\int_{1}^{2} \frac{\gamma}{g} \frac{\partial U}{\partial t} d x=\frac{\partial U}{\partial t} \int_{i}^{2} \frac{\gamma}{g} d x=\frac{\gamma_{m} L}{g} \frac{d U_{1}}{d t}
$$

Here

$$
\gamma_{m}=\frac{1}{L} \int_{1}^{2} \gamma d x
$$

Figure 12 shows an example of the distribution

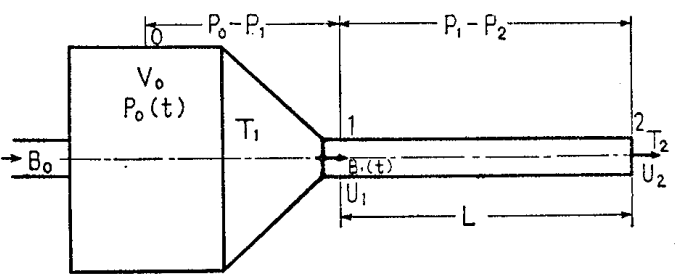

Fig. 11 Analytical model 
of axial mean temperature of the burnt gas in the combustion chamber. in this figure, the mean value of temperature in the combustion chamber is shown by two-dot chain line. This value is nearly in accordance with the burnt gas temperature at the outlet of combustion chamber. Therefore, the outlet temperature of the burnt gas can be regarded as the mean temperature of the burnt gas in the combustion chamber, and the value of $\gamma_{n}$ is determined from this value of temperature. Now, considering the steady state, Eq. (5) is given as follows :

$$
P_{1}-P_{2}=\lambda \frac{L\left(\gamma_{1} U_{1}\right)^{2}}{2 g d}\left(\frac{1}{\gamma}\right)_{m}+\frac{1}{g}\left(\gamma_{2} U_{2}^{2}-\gamma_{1} U_{1}^{2}\right)+\gamma_{m} L
$$

Here, $\lambda$ is determined from the experimental results of the pressure drop in the unburnt cold test. Using Eq. (2) and Eq. (5), The total pressure drop is obtained as follows :

Here,

$$
P_{0}-P_{2}=f \frac{\gamma_{1} U_{1}^{2}}{2 g}+\lambda \frac{L\left(\gamma_{1} U_{1}\right)^{2}}{2 g d}\left(\frac{1}{\gamma}\right)_{m}+\frac{1}{g}\left(\gamma_{2} U_{2}^{2}-\gamma_{1} U_{1}^{2}\right)+\gamma_{m} L+\frac{\gamma_{m} L}{g} \frac{d U_{1}}{d t}=\phi\left(U_{1}\right)+N \frac{d U_{1}}{d t}
$$

$$
\phi\left(U_{1}\right)=f \frac{\gamma_{1} U_{1}^{2}}{2 g}+\lambda \frac{L\left(\gamma_{1} U_{1}\right)^{2}}{2 g d}\left(\frac{1}{\gamma}\right)_{m}+\frac{1}{g}\left(\gamma_{2} U_{2}{ }^{2}-\gamma_{1} U_{1}{ }^{2}\right)+\gamma_{m} L, \quad N=\frac{\gamma_{m} L}{g}
$$

Let us introduce a new variable $H=P_{0}-P_{2}$. In this equation, $P_{2}$ has almost the same value as that of atmospheric pressure and its change with elapse of time is very small. Therefore, it may be assumed that the value of $P_{2}$ is constant. Accordingly,

$$
\frac{d P_{0}}{d t} \doteqdot \frac{d H}{d t}
$$

Substituting Eq. (10) into Eq. (1), $F \frac{d H}{d t}=B_{0}-A U_{1}$

From Eq. (8),

$$
H=\phi\left(U_{1}\right)+N \frac{d U_{1}}{d t}
$$

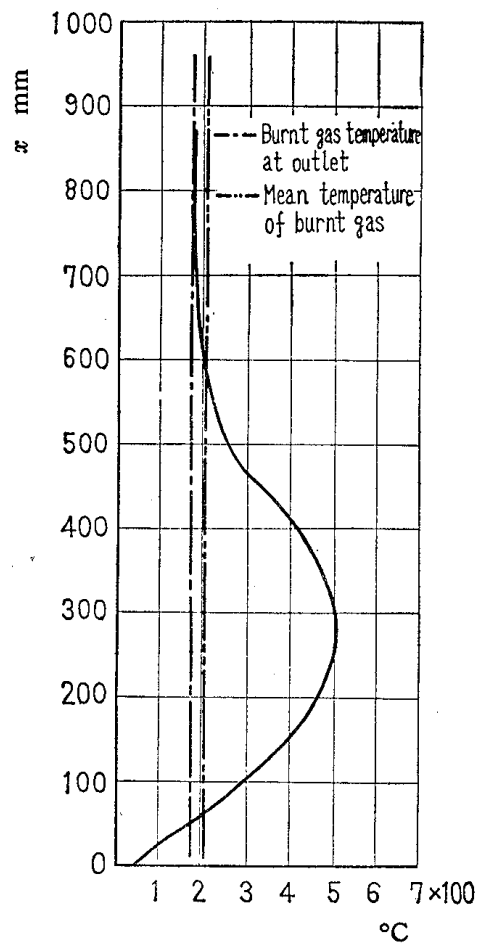

$d=49.5 \mathrm{~mm}, L=1.36 \mathrm{~m}, V_{0}=8.62 \times 10^{-2} \mathrm{~m}^{3}$

$M=6, Q_{f}=3.4 \mathrm{l} / \mathrm{m}, Q_{a_{1}}=33.9 \mathrm{l} / \mathrm{m}, \bar{U}_{1}=16.0 \mathrm{~m} / \mathrm{sec}$

Fig. 12 Example of the distribution of axial mean temperature of the burnt gas in the combustion chamber
Here, putting $U_{1}=\bar{U}_{1}+u, H=\bar{H}+h$ and $\phi\left(\bar{U}_{1}+u\right)=$ $\phi\left(\bar{U}_{1}\right)+\left[d \phi\left(\bar{U}_{1}\right) / d \bar{U}_{1}\right] u=\phi\left(\bar{U}_{1}\right)+\varphi(u)$, the following equations are obtained from Eq. (11)

$$
F \frac{d h}{d t}=\left(B_{0}-A \bar{U}_{1}\right)-A u=-A u
$$

and

$$
h=\varphi(u)+N \frac{d u}{d t}
$$

From Eq. (11)' and (12)', the governing equation in the system can be written as

$$
N \frac{d^{2} u}{d t^{2}}+a(u) \frac{d u}{d t}+\frac{A}{F} u=0
$$

Here, $a(u)=d \varphi(u) / d u=d \phi(\bar{U}) / d \bar{U}_{1}$, and it means the gradient of the characteristic curve which is determined by the experiment. Equation (13) is a differential equation of Van der Pol type. In this equation, it can be known that the oscillation in this problem is a self-excited oscillation and converges to a steady limit cycle with a constant period and a constant amplitude with the elaspe of time.

\subsection{Frequency}

Neglecting the second term of the left side of

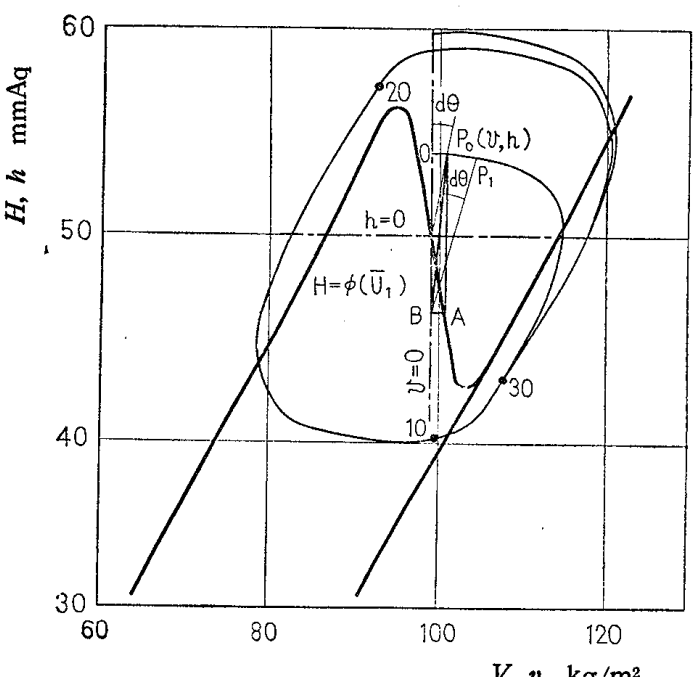

Numbers are corresponding to those in the analytical pattern as shown in Fig. 14

Fig. 13 Analysis on the phase-plane 
Eq. (13), the proper angular velocity is obtained,

$$
\omega=\sqrt{\frac{A}{N F}}=\sqrt{\frac{g}{\gamma_{m} L} \frac{\kappa \bar{P}_{0}}{V_{0}}} A=c \sqrt{\frac{\rho_{0}}{\rho_{m}} \frac{A}{L V_{0}}}
$$

Here, $c=\sqrt{\kappa \bar{P}_{0} / \rho_{0}}$ is the sonic velocity for the temperature at the inlet of combustion chamber. Hence, the proper frequency is

$$
\nu=\frac{c}{2 \pi} \sqrt{\frac{\rho_{0}}{\rho_{m}} \frac{A}{L V_{0}}}
$$

This value is corresponding to the Helmholtz frequency. The comparisons with the value calculated by Eq. (14) and the experimental results for various values of $L, V_{0}$ and $A$ are shown in Figs. 6, 7 and 8. Although $\rho_{m}$ is a mean value of density of the burnt gas in the combustion chamber, the value of $\rho_{2}$ or $\rho_{0}$ is used instead of $\rho_{m}$ in this case. The solution on the phase plane shown in the following section is also plotted in Fig. 8 by chain line. From these results, it is found that the analytical results are fairly well corresponding to the experimental results.

\subsection{Oscillation pattern}

Now, let's trace the motion of $u$ on this phase plane. Eliminating $d t$ from Eq. (11)' and (12)', we can get

$$
\frac{d h}{d u}=-\frac{A N}{F} \frac{u}{h-\varphi(u)}=\frac{1}{k}\left(-\frac{u}{h-\varphi(u)}\right)
$$

Here, $k=F / A N$.

Introducing $v=u / \sqrt{k}$ and then substituting it into Eq. (15),

$$
\frac{d h}{d v}=-\frac{v}{h-\varphi(u)}
$$

The analogous procedure to Lienard's method is used for the analysis of Eq. (16). Namely, the trajectory of the characteristic point can be described on $h-v$ plane as shown in Fig. 13 by combining the characteristic curve at the time of the burning and blow-out in Fig. 10 (b) and removing the origin of the coordinates to imaginary point. Now, the gradient $m_{1}$ of trajectory on the $h \cdot v$ plane is as follows from Eq. (16): $m_{1}=-v /\{h-\varphi(u)\}$. In Fig. 13, the coordinate of arbitrary point on the plane is marked as $\mathrm{P}_{0}(v, h)$. Drawing a line parallel to $h$-axis, the point where it intersects the characteristic curve is marked as A. Next, drawing a line parallel to $v$-axis from $\mathrm{A}$, the intersecting point at $h$-axis is marked as B. Now, the gradient of $\overline{\mathrm{BP}}_{0}$ is $m_{2}=\{h-\varphi(u)\} / v$. Therefore, $m_{1} m_{2}=-1$. Hence, the tangential line of the trajectory at the point is orthogonal to line $\overline{\mathrm{BP}_{0}}$. We can determine the point $P_{1}$ at a small distance from the point $P_{0}$ on the line $\overline{\mathrm{P}_{0} \mathrm{~T}}$ which is orthogonal to $\overline{\mathrm{BP}_{0}}$. $\quad \mathrm{By}$ applying the same manipulation, we can also determine the points $\mathrm{P}_{2}, \mathrm{P}_{3}$ and so on. In this way, the trajectory of the characteristic point on the $h-v$ plane can be obtained. Now, denoting $\angle \mathrm{P}_{0} \mathrm{BP}_{1}=$ $d \theta$, the following expression is obtained from Eq. (11)' and (16).

$$
d \theta=\frac{\overline{\mathrm{P}_{0} \mathrm{P}_{1}}}{\overline{\mathrm{BP}_{0}}} \frac{\sqrt{(d h)^{2}+(d v)^{2}}}{\sqrt{v^{2}+\{h-\varphi(u)\}^{2}}}=\frac{d h}{v}=-\omega d t \ldots
$$

Hence, we can draw the time scale on the trajectory. From Fig. 13 it is found that the deviation from the trajectory converges to a closed curve (limit cycle) with the elapse of time, and the oscillation with a constant period and a constant amplitude is formed.

Next, let us consider the motion of the characteristic point on the phase-plane more precisely. When the characteristic curve $\left[H=\phi\left(\bar{U}_{1}\right)\right]$ approaches the point of the flame necking and arrives at the region with a negative gradient of the curve, it jumps abruptly from the characteristic curve at the time of burning to that of blow-out. After jumping, the characteristic point goes down along the characteristic curve corresponding to the duration of blow-out while satisfying Eq. (16). Then it arrives at the point of $v=0$. At the point of $v=0, d h / d v$ is zero by Eq. (16). Therefore, the characteristic point jumps from the characteristic curve at the time of blow-out to that of burning. It goes up again along the characteristic curve of the duration of burning. Such a motion of the characteristic point can well explain both the fluctuation of the secondary air velocity and of flame. The trajectory of the phase-plane in Fig. 13 is rewritten as $u$ - $t$ diagram which is shown in the lower figure in Fig. 14. The upper diagram in Fig. 14 is an oscillogram of $U_{1}$ obtained in this experiment and coincides fairly well with the analytical results. Thus, it is found that the cause of generation about this oscillation is explained qualitatively in Fig. 14.

\section{Conclusions}

(1) The appearance of flame movement was observed in a simple combustion chamber using a

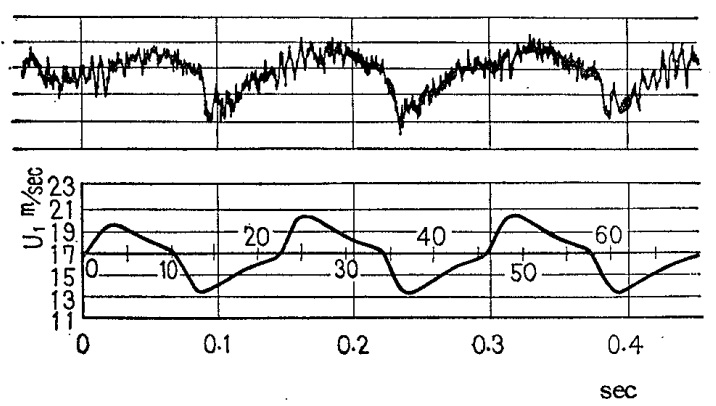

Upper: Experimental results of fluctuation of the secondary air velocity

Lower : Analytical pattern obtained by phase-plane $d=49.5 \mathrm{~mm}, L=1.36 \mathrm{~m}, V_{0}=8.62 \times 10^{-2} \mathrm{~m}^{3}, M=8$, $\bar{U}_{1}=16.5 \mathrm{~m} / \mathrm{sec}, Q_{f}=4.6 \mathrm{l} / \mathrm{m}, Q_{a_{1}}=56.0 \mathrm{l} / \mathrm{m}$,

Relative intensity of turbulence: $14.5 \sim 17.7 \%$

Fig. 14 Comparison between experimental result and analytical pattern 
gaseous fuel (propane). From results of some experiments, it was found that the low-frequency oscillation is generated in the vicinity of the flame necking. Considering the flame photographs and the record of a transitional state from the flame necking to the low-frequency oscillation, it was found that the generation of oscillation is mainly caused by the fluctuation of the secondary air velocity in the vicinity of the necking limit.

(2) The frequency of oscillation was slightly influenced by the flow rate of mixture, the secondary air velocity and the air-fuel ratio, and it was decided by the geometrical factors of the system.

(3) The experimental results of the pressure drop in the combustion chamber were well corresponding to the calculated values. It was confirmed that the pressure drop in the combustion chamber indicated a negative resistance with an increase of the secondary air velocity in the vicinity of the flame necking. Such a phenomenon can be explained by the fact that the value of accelerational loss is determined by the state of combustion.

(4) Taking into account the experimental results, the character of such a system could be represented by the differential equation of Van der Pol type.

(5) As the result of analysis, the limit cycle obtained on the phase-plane could well explain the fluctuation of the secondary air velocity and of flame, and the analytical value of frequencies. The oscillation pattern obtained by an analytical procedure. coincides well with the experimental results on oscillograms.

Thus, a consistent knowledge has been obtained for the various experimental results. However, we simply dealt with the character of the combustion. in the vicinity of flame necking as a problem due to the pressure drop. Taking into account the rela. tion between the character of combustion and the necking limit, more precise studies should be performed in future.

\section{References}

(1) L. Crocco and S. I. Cheng: Theory of Combustion Instabulity in Lipuid Propellant Rocket Motors, (1965), p, 25, Butterworths.

(2) M. W. Thring: 7 th Symp. Comb., (1959), p. 659.

(3) F. Mauss et al.: 10 th Symp. Comb., (1965), p. 1241

(4) T. Sato et al.: Bulletin of JSME, Vol. 6, No. 21 (1963), p. 69 .

(5) H. Iida: Report of Reserch Institute of Electricity Japan No. 22 (1963).

(6) Y. Tanasawa: 8th Symp. Comb., (1962), p. 1003.

(7) H. Nishimura: Trans. Japan Soc. Mech. Engrs., Vol. 29, No. 207 (1963), p. 1844 .

(8) H. Nishimura: Trans. Japan Soc. Mech. Engrs., Vol. 31, No. 229 (1565), p. 363.

(9) J. Barr: 4 th Symp. Comb., (1953), p. 765.

(10) S. Hayami : Bulletin of JSME, Vol. 6, No. 23 (1963) p. 549 . 